\title{
THE MULTIPLICITY OF EIGENVALUES
}

\author{
BY PETER D. LAX
}

There are many examples of first order $n \times n$ systems of partial differential equations in 2 space variables with real coefficients which are strictly hyperbolic; that is, they have simple characteristics. In this note we show that in 3 space variables there are no strictly hyperbolic systems if $n \equiv 2(4)$. Multiple characteristics of course influence the propagation of singularities. For a different context see Appendix 10 of [2].

$M$ denotes the set of all real $n \times n$ matrices with real eigenvalues. We call such a matrix nondegenerate if it has $n$ distinct real eigenvalues.

Theorem. Let $A, B, C$ be three matrices such that all linear combinations

$$
\alpha A+\beta B+\gamma C,
$$

$\alpha, \beta, \gamma$ real, belong to $M$. If $n \equiv 2(\bmod 4)$, then there exists $\alpha, \beta, \gamma$ real, $\alpha^{2}+$ $\beta^{2}+\gamma^{2} \neq 0$ such that (1) is degenerate.

RemarK 1. The theorem applies in particular to $A, B, C$ real symmetric.

REMARK 2. The theorem shows that first order hyperbolic systems in three space variables of the indicated order always have some multiple characteristics.

Proof. Denote by $N$ the set of nondegenerate matrices in $M$. The normalized eigenvectors $u$ of $N$ is $N$,

$$
N u_{j}=\lambda_{j} u_{j}, \quad\left|u_{j}\right|=1, \quad j=1, \ldots, n,
$$

are determined up to a factor \pm 1 .

Let $N(\theta), 0 \leqslant \theta \leqslant 2 \pi$, be a closed curve in $N$. If we fix $u_{j}(0)$, then $u_{j}(\theta)$ can be determined uniquely by requiring continuous dependence on $\theta$. Since $N(2 \pi)=N(\theta)$

$$
u_{j}(2 \pi)=\tau_{j} u_{j}(0), \quad \tau_{j}= \pm 1
$$

Clearly

(i) Each $\tau_{j}$ is a homotopy invariant of the closed curve.

(ii) Each $\tau_{j}=1$ when $N(\theta)$ is constant.

Suppose now that the theorem is false; then

$$
N(\theta)=\cos \theta A+\sin \theta B
$$

Received by the editors June 4, 1981.

1980 Mathematics Subject Classification. Primary 15A18, 57R99, 35L40.

(c) 1982 American Mathematical Society 0273-0979/81/0000-0142/\$01.50 
is a closed curve in $N$. Note that $N(\pi)=-N(0)$; this shows that

$$
\begin{aligned}
& \lambda_{j}(\pi)=-\lambda_{n-j+1}(0) \text { and } \\
& u_{j}(\pi)=\rho_{j} u_{n-j+1}(0), \quad \rho_{j}= \pm 1 .
\end{aligned}
$$

Since the ordered basis $\left\{u_{1}(\theta), \ldots, u_{n}(\theta)\right\}$ is deformed continuously, it retains its orientation. Thus the ordered bases

$$
\left\{u_{1}(0), \ldots, u_{n}(0)\right\} \quad \text { and } \quad\left\{\rho_{1} u_{n}(0), \ldots, \rho_{n} u_{1}(0)\right\}
$$

have the same orientation. For $n \equiv 2(\bmod 4)$, reversing the order reverses the orientation of an ordered base; this proves that

$$
\prod_{1}^{n} \rho_{j}=-1
$$

This implies that there is a value of $k$ for which

$$
\rho_{k} \rho_{n-k+1}=-1 \text {. }
$$

Next we observe that $N(\theta+\pi)=-N(\theta)$; it follows from this that $\lambda_{j}(\theta+\pi)=$ $-\lambda_{n-j+1}(\theta)$ and by (4) that

$$
u_{j}(2 \pi)=\rho_{n-j+1} u_{n-j+1}(\pi) .
$$

Combining this with (4) we get that $\tau_{j}=\rho_{j} \rho_{n-j+1}$. By (5), $\tau_{k}=-1$; this shows that the curve (3) is not homotopic to a point.

Suppose that all matrices of form (1), $\alpha^{2}+\beta^{2}+\gamma^{2}=1$, belonged to $N$. Then since the sphere is simply connected the curve (4) could be contracted to a point, contradicting $\tau_{k}=-1$.

See [1] for related matters.

AdDed in Proof. S. Friedland, J. Robbin and J. Sylvester have proved the theorem for all $n \equiv \pm 2, \pm 3, \pm 4(\bmod 8)$, and have shown it false for $n=0$, $\pm 1(\bmod 8)$. They have further results involving linear combinations of more than 3 matrices.

\section{REFERENCES}

1. F. John, Restriction on the coefficients of hyperbolic differential equations, Proc. Nat. Acad. Sci. U. S. A. 74 (1977), 4150-4151.

2. V. I. Arnold, Math. methods in classical mechanics, Lecture Notes in Math., vol. 60, Springer-Verlag, Berlin and New York, 1978.

COURANT INSTITUTE OF MATHEMATICAL SCIENCES, NEW YORK UNIVERSITY, NEW YORK, NEW YORK 10012 